\title{
Quantum walk on the line as an interference phenomenon
}

\author{
Peter L. Knight, ${ }^{*}$ Eugenio Roldán, ${ }^{\dagger}$ and J. E. Sipe \\ Optics Section, Blackett Laboratory, Imperial College London, London SW7 2AZ, United Kingdom
}

(Received 30 April 2003; published 27 August 2003)

\begin{abstract}
We show that the coined quantum walk on a line can be understood as an interference phenomenon, can be classically implemented, and indeed already has been. The walk is essentially two independent walks associated with the different coin sides, coupled only at initiation. There is a simple analogy between the evolution of walker positions and the propagation of light in a dispersive optical fiber.
\end{abstract}

DOI: 10.1103/PhysRevA.68.020301

PACS number(s): 03.67.Lx, 05.40.Fb

The quantum random walk (QW) was first proposed ten years ago by Aharonov, Davidovich, and Zagury [1] as the quantum analog of the classical random walk (RW). QWs are receiving much attention [2-14]: as some problems are best solved in classical computation with algorithms based on RWs, it is expected that this type of problems could be solved even faster in a quantum computer. Preliminary investigations focused on the nature of the QWs themselves. For example, Kempe [4] has shown that the hitting time of the discrete QW from one corner of an $N$-bit hypercube to the opposite corner is polynomial in the number of steps, $n$, while it is exponential in $n$ in the classical case. Subsequently Shenvi, Kempe, and Whaley [5] showed that a QW can perform the same tasks as Grover's search algorithm, and Childs et al. [6] introduced an algorithm for crossing a special graph exponentially faster that can be done with a classical RW. Kempe [14] has recently reviewed the field.

In the classical RW on the line, the "walker" (the particle or system performing the RW) randomly takes one step to the right or to the left depending on the result of tossing a coin. After $n$ steps, the probability of finding the walker at a distance $m$ from the origin is given by the binomial distribution, a Gaussian for large $n$ with a standard deviation $\sigma$ $=\sqrt{n}$. In the $\mathrm{QW}$, the role of the coin is played by a qubit (as, e.g., a two-level atom or a spin- $\frac{1}{2}$ particle). As its classical counterpart, the quantum walker moves to the right or to the left depending on the internal state of the qubit. After each displacement, the state of the qubit is set to a superposition state by means of a suitable unitary transformation, typically a Hadamard, that plays the role of the toss of the coin in the RW. Yet the QW is not a random walk, as its time evolution is completely deterministic. The probability distribution in the quantum case is very different from the classical one: it resembles the Airy function (Fig. 1) and has a standard deviation that is linear with $n$. This is the discrete time QW that should be distinguished from the continuous time QW $[2,14]$, which we will not consider here.

\footnotetext{
*Email address: p.knight@imperial.ac.uk

†Permanent address: Departament d'Òptica, Universitat de València, Dr. Moliner 50, 46100-Burjassot, Spain. Email address: eugenio.roldan@uv.es

*Permanent address: Department of Physics, University of Toronto, Toronto M5S 1A7, Canada. Email address: sipe@physics.utoronto.ca
}

Possible implementations of the QW have been proposed by a number of authors [10-12]. Here we show that a classical implementation of the QW is possible, in analogy with other processes usually associated with quantum computing [15-18]. Indeed, we point out that a classical implementation very similar to the one we are proposing has actually been implemented by Bouwmeester et al. [19], in the context of the optical Galton board, without the authors explicitly noting this. Other classical (interferometric) implementations of the QW have been proposed recently $[8,13]$, but in them the number of necessary optical elements grows quickly with the number of steps in the QW, something that does not occur in our scheme. Finally, by reexamining the difference equations for the walker we show that the nature of propagation is simpler than has been previously appreciated.

In our classical approach the role of the walker is played by the frequency of a light field, and the role of the coin is played by its polarization state. The light field

$$
\vec{E}=\sum_{m=-l}^{l} \vec{E}_{m} \exp \left[i\left(\omega_{0}+m \bar{\omega}\right) t-i k_{m} z\right]+\text { c.c. }
$$

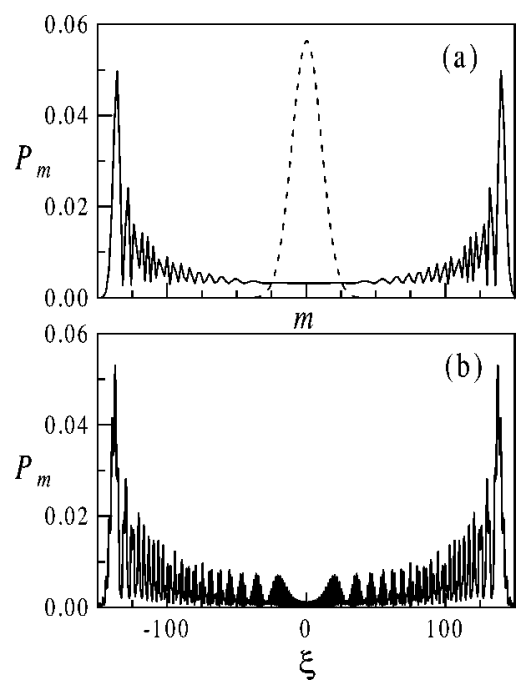

FIG. 1. (a) Probability distribution for $n=200$ for both the classical (dashed) and quantum (continuous) random walks. The initial conditions chosen for calculating the QW were $R_{0,0}=1 / \sqrt{2}$ and $L_{0,0}=i / \sqrt{2}$, see Eqs. (4) and (5). Notice that the quantum $P_{m}$ is null for odd $m$ at odd $n$. We have represented only nonzero values. (b) Continuous limit of the QW as given by Eq. (13) for $\alpha=0.4$ and $t=200$ with the same initial conditions as in (a). 
( $\omega_{0}$ is the carrier frequency and $\bar{\omega}$ is the frequency difference between successive frequency components) can be represented by the abstract state

$$
\left.\left.\mid \psi)=\sum_{m=-l}^{l}\left[R_{m} \mid m, x\right)+L_{m} \mid m, y\right)\right],
$$

where $R_{m} \equiv \hat{x} \cdot \vec{u}_{m}$ and $L_{m} \equiv \hat{y} \cdot \vec{u}_{m} \quad\left(\vec{u}_{m}=\vec{E}_{m} /\left|\vec{E}_{m}\right|\right)$ and $\sum_{m=-l}^{l}\left[\left|R_{m}\right|^{2}+\left|L_{m}\right|^{2}\right]=1$; the "basis vectors" $\left.\mid m, c\right)$ label the frequency and polarization, with $c=x, y$; we associated $x(y)$ with the coin head (tail).

To implement the walk, we require a unitary operator that performs

$$
\left.\left.\hat{V} \mid m, \begin{array}{l}
x \\
y
\end{array}\right)=\mid m \pm 1, \begin{array}{l}
x \\
y
\end{array}\right) .
$$

The operation $\hat{V}$ can be physically implemented, e.g., with an electrooptic modulator (EOM) to which a linearly time dependent voltage is applied in such a way that the $x(y)$ polarization component of the field frequency component $\left(\omega_{0}\right.$ $+m \bar{\omega})$ will see its frequency increased (decreased) by an amount $\bar{\omega}$.

After each jump in the frequency of the field, a Hadamard transformation

$$
\left.\left.\left.\hat{H} \mid m, \begin{array}{l}
x \\
y
\end{array}\right)=\frac{1}{\sqrt{2}}[\mid m, x) \pm \mid m, y\right)\right]
$$

has to be implemented. This can be done optically by means of a half-wave plate (HWP) with its fast axis forming an angle $\pi / 8$ with respect to the $\hat{x}$ axis $[15,16]$. Finally, the QW is implemented by the repeated action on the state of the operator $\hat{H} \hat{V}$, i.e., after $n$ iterations $\left.\mid \psi(n))=[\hat{H} \hat{V}]^{n} \mid \psi(0)\right)$, which can be written as

$$
\begin{gathered}
\left.\left.\mid \psi(n))=\sum_{m=-n}^{+n}\left[R_{m, n} \mid m, x\right)+L_{m, n} \mid m, y\right)\right], \\
R_{m, n}=\frac{1}{\sqrt{2}}\left(R_{m-1, n-1}+L_{m+1, n-1}\right), \\
L_{m, n}=\frac{1}{\sqrt{2}}\left(R_{m-1, n-1}-L_{m+1, n-1}\right),
\end{gathered}
$$

where $R_{m, 0}=L_{m, 0}=0$ if $m \neq 0$ and $R_{m,-1}=L_{m,-1}=0 \quad \forall m$. These are the standard QW equations. Finally, the intensity of each frequency component of the light field, which is the optical analog of the probability of finding the walker at position $m$ at iteration (time) $n$, is given by $P_{m, n}=\left|R_{m, n}\right|^{2}$ $+\left|L_{m, n}\right|^{2}$, which is represented in Fig. 1 .

In order to implement $n$ steps the best option is to introduce the described elements in an optical cavity, Fig. 2. The cavity imposes a constraint that the optical frequencies must fit within its set of eigenfrequencies. Thus, the time dependent electric field applied to the EOM and the cavity length must be adjusted in such a way that the frequency shift $\bar{\omega}$ $=f \omega_{\mathrm{FSR}}$ with $\omega_{\mathrm{FSR}}$ being the cavity free spectral range and $f$

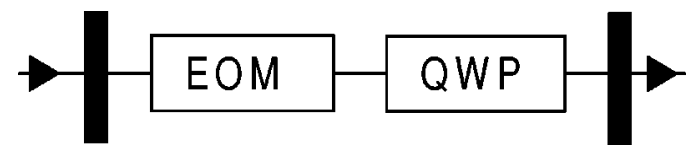

FIG. 2. Scheme for the optical implementation of the QW in a Fabry-Perot cavity. The electro-optic modulator (EOM) shifts the field frequency up or down in $\bar{\omega} / 2$ depending on its polarization, and a quarter-wave plate (QWP) with its axis forming an angle $\pi / 8$, with respect to the $x$ axis, performs the Hadamard transformation (notice that light passes twice through each intracavity element every round-trip).

being an integer number. Consider nevertheless that a light pulse with a spectral width $\Delta \omega$ is initially injected in the cavity. Although in such a case $f$ does not need to be an integer, in order to perform a step of the QW at each cavity roundtrip, the step size $\bar{\omega}$ must be large enough to avoid significant overlap between the spectra of the displaced pulses; thus the frequency steps are well resolved.

The experiment of Bouwmeester et al. [19] can be seen as a realization of the QW very similar to that proposed here. These researchers proposed and studied, both theoretically and experimentally, an optical implementation of the Galton Board (the quincunx). What they actually implement is a grid of Landau-Zener crossings through which a light beam propagates, and concentrate on the study of recurrences in the light spectrum. A simplified version of their experimental device is that represented in Fig. 2, but with the QWP replaced by a second EOM with its axis rotated $\pi / 4$ with respect to the first EOM, which introduces a dephasing between the two polarization components. Although this unitary operation does not correspond to a Hadamard transformation, it can be shown that it leads to an essentially identical QW [5] (details to be reported elsewhere). The main difference with our proposal is that the frequency shift introduced by the EOM is smaller than $\omega_{\mathrm{FSR}}$ and then each step in the QW takes several cavity round-trips. In Fig. 6 of Ref. [19] the QW is clearly seen. Bouwmeester et al. [19] considered this case as a demonstration of the coherence quality of their system, and did not note its significance to QWs; their focus on the observation of recurrences in the spectrum led them to study other aspects of their system.

Let us now reexamine the linear difference equations (4) and (5). They admit a formal solution that has been studied from a number of points of view, usually with a focus on identifying its asymptotic behavior for large $n[3,9,14]$ as it allows for the extraction of much information. Nevertheless the formal solutions presented to date do not rely explicitly on a crucial feature of Eqs. (4) and (5), which we now explain, that greatly simplifies a physical understanding of their solution. A little algebra reveals that the solutions $R_{m, n}$ and $L_{m, n}$ of Eqs. (4) and (5) also satisfy

$$
a_{m, n+1}=a_{m, n-1}+\frac{1}{\sqrt{2}}\left[a_{m-1, n}-a_{m+1, n}\right], \quad a=R, L .
$$

This is a remarkable equation, since it demonstrates a dynamical independence of the evolution of the two coin states $R$ and $L$. Thus there are two essentially independent walks, 
coupled only by the first step that links $a_{m, 1}$ to $a_{m, 0}$. After that, the two walks can be studied independently of each other.

The most naive continuous limit of Eq. (6) would involve a first derivative with respect to time and a first derivative with respect to space, and would suggest waves propagating only towards $+\infty$ for both $R$ and $L$, in apparent violation of the symmetry of the problem. But this is too simplistic, given that for both $R$ and $L$ one can look for solutions of the form

$$
a_{m, n}=A_{m, n}^{+}+(-1)^{n} A_{m, n}^{-},
$$

where $A_{m}^{ \pm}$satisfy

$$
A_{m, n+1}^{ \pm}-A_{m, n-1}^{ \pm}= \pm \frac{1}{\sqrt{2}}\left[A_{m-1, n}^{ \pm}-A_{m+1, n}^{ \pm}\right]
$$

restoring the symmetry. Of course, there is not a unique specification of the $A_{m}^{ \pm}$in terms of the fundamental $a_{m}$, since

$$
\begin{aligned}
& a_{m, 0}=A_{m, 0}^{+}+A_{m, 0}^{-}, \\
& a_{m, 1}=A_{m, 1}^{+}-A_{m, 1}^{-} .
\end{aligned}
$$

The specification of the $a_{m, 0}$ and $a_{m, 1}$, which completely specifies the initial conditions required to solve Eq. (6), does not suffice to determine the initial conditions $A_{m, 0}^{ \pm}$and $A_{m, 1}^{ \pm}$ required for the solution of Eq. (8) uniquely. Nonetheless, it is possible to rigorously develop the solution of Eqs. (6) in terms of the fields $A_{m, n}^{ \pm}$; this we defer to a later publication. The point we wish to stress here is that in the limit of $A_{m, n}^{ \pm}$ that are slowly varying in $n$ and $m$ we can introduce continuous functions $A^{ \pm}(x, t)$ and understand Eq. (8) as the discretization of the differential equation

$$
\begin{aligned}
& \sum_{k=0}^{\infty} \frac{(\Delta t)^{2 k+1}}{(2 k+1) !} \frac{\partial^{2 k+1}}{\partial t^{2 k+1}} A^{ \pm}(x, t) \\
& \quad=\mp \frac{1}{\sqrt{2}} \sum_{k=0}^{\infty} \frac{(\Delta x)^{2 k+1}}{(2 k+1) !} \frac{\partial^{2 k+1}}{\partial x^{2 k+1}} A^{ \pm}(x, t),
\end{aligned}
$$

where $\Delta t$ and $\Delta x$ denote the temporal and spatial increments, respectively. Keeping only the first two terms and approximating the third derivative in time using the equation at the lowest order, we obtain

$$
\frac{\partial}{\partial \tau} A^{ \pm}(\xi, \tau)=\mp \frac{1}{\sqrt{2}}\left[\frac{\partial}{\partial \xi}+\frac{1}{12} \frac{\partial^{3}}{\partial \xi^{3}}\right] A^{ \pm}(\xi, \tau),
$$

where $\tau=t / \Delta t$ and $\xi=x / \Delta x$. In this slowly-varying approximation conditions (9) can be approximated as

$$
A^{ \pm}(m(\Delta x), 0) \approx A_{m, 0}^{ \pm}=\frac{1}{2}\left(a_{m, 0} \pm a_{m, 1}\right),
$$

where we have made use of Eq. (7) and assumed that $a_{m, 1}$ $=A_{m, 1}^{+}-A_{m, 1}^{-} \approx A_{m, 0}^{+}-A_{m, 0}^{-}$. Thus in this limit Eq. (12) pro- vides the initial conditions for Eq. (11) and those equations can be solved by Fourier analysis. There are then two fields $A^{ \pm}(\xi, \tau)$ that can be associated with each side of the coin. This feature persists when the rigorous solution is constructed in this terminology, where there is a (temporal) "ferromagnetic" field $A_{m, n}^{+}$and an "antiferromagnetic" field $(-1)^{n} A_{m, n}^{-}$for each coin side.

Returning to Eq. (11) we make use of Eqs. (12) and take as initial conditions $A^{ \pm}(\xi, 0)=a_{0,0} G(0) \pm a_{-1,1} G(-1)$ $\pm a_{1,1} G(1)$, with $G\left(\xi_{0}\right)=\mathcal{N} \exp \left[-\left(\xi-\xi_{0}\right)^{2} /(2 \alpha)^{2}\right]$ and $\mathcal{N}$ a normalization factor; here we assume that Eq. (11) is only correct for the long-wavelength components by taking an initial condition that "smears out" the lower-wavelength components. The solution is easily found analytically $[20,21]$ for $A^{ \pm}(\xi, \tau)$, and we can write the final result (up to a normalization factor) for both $R$ and $L$ as $a(\xi, \tau)=A^{+}(\xi, \tau)+$ $(-1)^{n} A^{-}(\xi, \tau)$, with

$$
\begin{aligned}
A^{ \pm}(\xi, \tau)= & a_{0,0} Z( \pm \xi, \tau) \pm a_{-1,1} Z\left( \pm\left(\xi+\xi_{0}\right), \tau\right) \\
& \pm a_{1,1} Z\left( \pm\left(\xi-\xi_{0}\right), \tau\right), \\
Z(\xi, \tau)= & \frac{2 \pi}{\mathcal{B}^{1 / 3}} \exp \left(\frac{3 \mathcal{A B C}+2 \mathcal{C}^{3}}{3 \mathcal{B}^{2}}\right) A_{i}\left(\frac{\mathcal{A B}+\mathcal{C}^{2}}{\mathcal{B}^{4 / 3}}\right),
\end{aligned}
$$

where $\mathcal{A}=\xi-\tau / \sqrt{2}, \mathcal{B}=\tau /(4 / \sqrt{2}), \mathcal{C}=\alpha^{2}$ and $A_{i}(x)$ is the Airy function [21]; the $R$ and $L$ solutions differ only in the different values of $a_{m, 0}$ and $a_{m, 1}$ appearing in Eq. (13). The appearance of Airy functions in the full solutions of Eqs. (4) and (5) [9] can thus be understood as associated with the form of Eq. (11), which workers in fiber optics will recognize as the classical equation for the propagation of light in a fiber with no group velocity dispersion but a third-order dispersion term. The linear dependence of the standard deviation on $n$ arises, of course, simply because of this propagation. Solution (13) is represented in Fig. 1(b) for $\alpha=0.4$, and the similarity with the QW in Fig. 1(a) is clearly apparent.

In conclusion, we have shown that the QW along a line can be simulated in a purely classical implementation, involving nothing more than wave interference of electromagnetic fields. And, indeed, it has in fact already been simulated in the laboratory in the work of Bouwmeester et al. [19].

Further, this classical nature of the propagation is perhaps not surprising. After all, the standard QW is a generalization of the quantum-mechanical problem of a spinless particle with hopping amplitudes between sites, familiar from solidstate physics if the time variable is continuous. That latter problem, which gives a simple Schrödinger equation in its continuum limit, is clearly classical in nature of its propagation, as attested to by the appearance of the Schrödinger equation in classical beam propagation problems. The generalization involved in concocting the standard QW problem is the inclusion of a spin variable. What we have shown here is that this generalization does not affect the dynamics in an essential way. Except for an initial coupling in the first two time steps, the evolutions of the amplitudes associated with the two sides of the coin proceed independently. 
This work was supported in part by the UK Engineering and Physical Sciences Research Council and the European Union. E.R. acknowledges financial support from the Ministerio de Educación, Cultura y Deportes of the Spanish Gov- ernment (Grant No. PR20002-0244). J.E.S. acknowledges financial support from the Natural Sciences and Engineering Research Council of Canada. We gratefully acknowledge fruitful discussions with V. Kendon.
[1] Y. Aharonov, L. Davidovich, and N. Zagury, Phys. Rev. A 48, 1687 (1993).

[2] E. Farhi and S. Gutmann, Phys. Rev. A 58, 915 (1998).

[3] A. Nayak and A. Vishwanath, e-print quant-ph/0010117.

[4] J. Kempe, e-print quant-ph/0205083.

[5] N. Shenvi, J. Kempe, and K. B. Whaley, Phys. Rev. A 67, 052307 (2003).

[6] A. M. Childs, R. Cleve, E. Deotto, E. Farhi, S. Gutmann, and D. A. Spielman, e-print quant-ph/0209131.

[7] V. Kendon and B. Tregenna, Phys. Rev. A 67, 042315 (2003).

[8] M. Hillery, J. Bergou, and E. Feldman, e-print quant-ph/0302161.

[9] H. A. Carteret, M. E. H. Ismail, and B. Richmond, e-print quant-ph/0303105.

[10] B. C. Travaglione and G. J. Milburn, Phys. Rev. A 65, 032310 (2002).

[11] W. Dür, R. Raussendorf, V. M. Kendon, and H.-J. Briegel, Phys. Rev. A 66, 052319 (2002).
[12] B. C. Sanders, S. D. Bartlett, B. Tregenna, and P. L. Knight, Phys. Rev. A 67, 042305 (2003).

[13] H. Jeong, M. Paternostro, and M. S. Kim, e-print quant-ph/0305008.

[14] J. Kempe, Contemp. Phys. 44, 307 (2003).

[15] R. J. C. Spreeuw, Phys. Rev. A 63, 062302 (2001).

[16] N. J. Cerf, C. Adami, and P. G. Kwiat, Phys. Rev. A 57, R1477 (1998).

[17] P. G. Kwiat, J. R. Mitchell, P. D. D. Schwindt, and A. G. White, J. Mod. Opt. 47, 157 (2000).

[18] N. Bhattacharya, H. B. van Linden van den Heuvell, and R. J. C. Spreeuw, Phys. Rev. Lett. 88, 137901 (2002).

[19] D. Bouwmeester, I. Marzoli, G. P. Karman, W. Schleich, and J. P. Woerdman, Phys. Rev. A 61, 013410 (2000).

[20] M. Miyagi and S. Nishida, Appl. Opt. 18, 678 (1979).

[21] Handbook of Mathematical Functions, edited by M. Abramowitz and I. A. Stegun (Dover, New York, 1970), pp. $446-475$. 